\title{
METAPLASTIC BREAST CARCINOMA: CASE SERIES
}

Fergus Tomás Rocha de Oliveira', Rafael Everton Assunção Ribeiro da Costa', Eduarda Norberto Siqueira', Ana Lúcia Nascimento Araújo², Sabas Carlos Vieira³

${ }^{1}$ Universidade Estadual do Piauí - Teresina (PI), Brazil.

${ }^{2}$ Hospital São Marcos - Teresina (PI), Brazil.

${ }^{3}$ Oncocenter - Teresina (PI), Brazil.

Introduction: Metaplastic breast carcinoma (MBC) is understood as a heterogeneous group of malignant tumors, which exhibits the transformation of part or its entire glandular carcinomatous component into a nonglandular component by means of epithelial and mixed mesenchymal differentiation. The $\mathrm{MBC}$ is a rare and aggressive breast cancer, accounting for approximately $1 \%$ of all breast tumors that have a worse prognosis. The aim of this study was to describe four cases of $\mathrm{MBC}$, analyzing the progression of patients in a mean follow-up of 25 months (11-43 months). Case series: All patients were female. The mean age of the four cases of the series was 50 years (40-61 years). All patients had tumors that were histologically classified as metaplastic carcinoma. The mean tumor size of the series was $8.3 \mathrm{~cm}(2.2-15.5 \mathrm{~cm})$. Two patients had the angiolymphatic invasion, and none had the perineural invasion. In three cases, there was an axillary node involvement. None of the cases had metastasis at the time of diagnosis. All patients underwent surgical treatment. Concerning complementary treatment, all patients underwent chemotherapy, three underwent radiation therapy, and two received hormone therapy. Three patients had systemic recurrence with metastases (i.e., all progressed to death). The study was approved by a Research Ethics Committee, under CAAE No 30154720.0.0000.5209. Conclusion: With a mean follow-up of 25 months (11-43 months), three out of four cases (75\%) presented systemic recurrence with metastases (i.e., all culminating in death), and one patient is alive without evidence of malignancy.

Keywords: Medical Records; Breast Neoplasms; Recurrence; Neoplasm Metastasis. 